\title{
Physics in Turkey
}

\author{
Erdal Inönü, Istanbul
}

IBogaziçi University,

Chairman of the Local Organizing Committee of the 5th EPS General Conference)

Leaving aside activities in medieval science, it can be said, without much exaggeration, that productive research in "European" physics started in Turkey in the 1930's, although the break with the medieval tradition represented by the Islamic schools began already in the second half of the eighteenth century. Schools of engineering (and later of medicine) were then opened where elements of European science developed over the previous two hundred years were taught. The transition from medieval to post Renaissance science was nevetheless, rather slow and difficult, taking about 150 years. All through this time, the emphasis was on teaching (or to use modern terminology, on the transfer of knowledge) through the translation of European textbooks and with the occasional help of visiting professors coming from foreign universities. Some of these visitors stayed for several years in Turkey (mostly in Istanbul) and engaged in research in various fields of science such as geology, mathematics, mechanics and chemistry. Also a few Turkish scientists contributed original articles to European science journals during this period. However, these individual efforts were not sufficient to create a sustained research activity in physics. Such a development only occurred after the Reform of Istanbul University in 1933.

\section{Development Pattern}

Figure 1(a), shows roughly the growth in the number of research articles written by physicists (Turkish or foreign) working in Turkey in the period 1936-1976. For the first fifteen years, the output remained restricted to about five papers per year, most of which were due to visiting professors (from France, Germany, Switzerland) and to young Turkish physicists returning from European universities after the completion of their doctoral studies. The second world war caused an understandable drop and then after 1948, the research production grew exponentially, chiefly as the result of the work of graduate students and young Ph.D.s at the universities of Istanbul (mainly) and Ankara. The growth continued up to 1975 which is the final year for which data exist, when the yearly output was $30-40$ papers; it is now estimated to be about $60-70$ papers. The Figure indicates, in addition, two periods of diminished research intensity during the past 35 years. The first covers roughly the years 1957-62 and reflects the economic hardships suffered by the universities along with the rest of the country at that time; the second drop observed in the period 1966-69 is a local effect and corresponds to a shift of the main research activity from Istanbul to Ankara. (The rise generated by new institutions was not rapid enough to compensate for the decline in the old institutions.)

To appreciate better, various aspects of physics research connected with Turkey, one should also consider the two histogrammes given in Figs. 1 (b) and (c). Fig. 1 (b) represents the total output from research carried out in Turkey or abroad by physicists affiliated in one way or another to Turkish institutions. The ordinates in (b) are obtained by adding to the corresponding figures in (c), the number of papers published by Turkish doctoral students in foreign universities and by staff members of Turkish universities working temporarily abroad, as visiting researchers. The pattern indicated by (b), roughly parallels that of (a), (although at a higher level) with the exception of the drop around 1967. Fig. 1(c), on the other hand which shows a different growth pattern, represents the research production of physicists of Turkish origin who seem to have established themselves permanently abroad. This production, which can be taken to indicate the effect of the "brain drain", grew rapidly after 1955 and has reached in 1975 about the same level as the number of papers produced on the basis of research done in Turkey although the actual number of physicists involved is much less.

\section{Lines of Research}

At present, research on pure and applied physics is conducted in Turkey at about 20 major institutions (universities and Government research institutes) by some 600 career physicists and students. In order to give a flavour of the subjects treated, I shall mention some of the work carried out by the main groups. To date, specific units for high energy (elementary particles and general relativity), space sciences, condensed matter and magnetic resonance have been organized under the aegis of the Turkish National Research Council (T.B.T.A.K.). Each unit holds its own annual or biannual symposium for the discussion of recent developments and the presentation of original contributions. In addition, there is a general meeting every year organized either by T.B.T.A.K. or the Turkish Physical Society.

\section{High Energy}

The last colloquia of the high energy unit featured contributions on classical Yang-

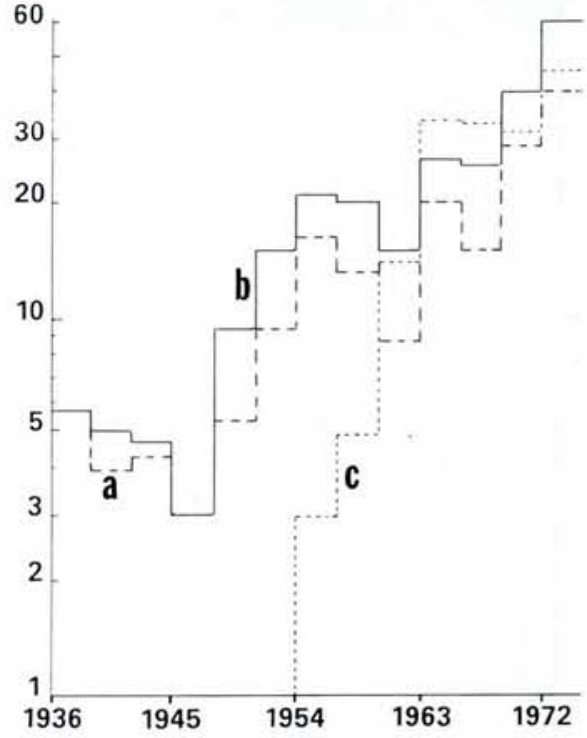

Fig. 1(a) (dashed line) - Original articles based on physics research in Turkey. (All histograms expressed as three year averages.)

$1(b)$ (solid line) - Original articles published by physicists associated with Turkish institutions, based on research in Turkey or abroad.

1(c) (dotted line) - Original articles by physicists of Turkish origin living abroad.

Mills solutions, instantons and merons, in particular gravitational instantons, grand unified theories based on exceptional groups, exact non-trivial solutions in super gravity, a recent proof of the no-hair conjecture for black holes in super gravity, model calculations in two-dimensional theories, QCD phenomenology, weak interactions and new particles, Hydrogenium and Hydrogenium-lon results of the CERN (emulsion) experiment for the observation of charmed particles, a phenomenological analysis of high-energy cross sections with predictions on the observations of new quarks. Obviously, the high energy work is predominantly theoretical. Our main opportunity for participation in experimental high energy research has, so far, lain in collaborating in international projects organized by CERN. A further collaboration is now planned with NAL.

\section{Space Sciences}

This unit brings together groups which have been working on traditional topics in astronomy and astrophysics at various university and Government observatories, with those which have been more recently formed in a few physics departments to deal with new developments in astrophysics. Among the lines of research one may 
mention are: multi-colour photometry of stars, observations on the constitution of star atmospheres, model calculations on the structure and evolution of stars, solar physics, $\mathrm{X}$-and $\gamma$-ray astronomy. Good collaboration with institutions in Europe or the USA has been established for carrying out the required observations. To mention some examples: there are long standing cooperation arrangements between the universities of Istanbul and Paris; the universities of Ankara, Istanbul, Ege (in Izmir) and Trieste; the Middle East Technical University (in Ankara), Cukurova University (in Adana) and NASA, ESA (e.g. for the analysis of the analysis of the $\gamma$-ray telescope data from SAS- 2 and COSB). An example of local astrophysical observations carried out in the physics departments is the use of the method of atmospheric scintillations in the search for supernovae or X-ray double stars. As yet, no new supernova has been discovered in this attempt, but an unexpected byproduct has been the observation of some interesting fast atmospheric pulses of apparently local origin. Another project of collaboration with NASA is in the field of ionospheric physics and involves the analysis of electron density data from the polar orbiting satellites Ariel 3 and 4 .

\section{Condensed Matter}

This unit deals at the moment mostly with solid state physics. Laboratories have been established in every major institution and the main effort in experimental physics is concentrated in this field. To give an idea of the main lines of research, let me refer to the programme of the latest symposium organized by this unit. It featured invited talks on ultrasonic waves in solids (in particular the use of ultrasonics to study changes in elastic properties caused by radiation), developments in crystal structure studies for organic crystals, the problem of phase and direct methods of structural analysis, profile analysis in the powder diffraction method, electronic properties of semiconductor surfaces, point defects in semiconductors, problems associated with Schottky diodes, resonance electron tunnelling in Schottky barriers, amorphous semiconductors, solar cells, colour centres in insulators, magnetic dilute alloys.

\section{Magnetic Resonance}

This unit represents a collaboration effort between several departments of physics and chemistry. It deals with structure studies conducted with various experimental resonance methods such as EPR, ESR, NMR, Relaxation, DNP, ENDOR and NQR. Among the lines of research, we may mention are studies of NMR hyperfine structure, asymmetrical properties in heterocyclic compounds, dynamical nuclear polarization in liquids, spin dynamics in liquid crystals, polymers and molecular solids, relaxation time measurements (in asphalt

\section{L'ÉCOLE POLYTECHNIQUE FÉDÉRALE DE LAUSANNE}

met au concours un poste de

\section{PROFESSEUR EN OPTOÉLECTRONIQUE}

à l'Institut de physique appliquée de son Département de physique.

Délai d'inscription: 31 octobre 1981.

Entrée en fonctions: 1er septembre 1982 ou à convenir.

Les personnes intéressées voudront bien demander le dossier relatif à ce poste à la:

Direction Administrative de I'Ecole Polytechnique Fédérale de Lausanne,

33. Avenue de Cour, CH-1001 Lausanne, Suisse. and asphaltines, e.g.), combined ESRNMR in biological systems.

Nuclear Physics

This is another field in which participation in topical experiments can only be realized outside the country. On the other hand, there are about twenty nuclear theorists in various departments and institutes who are actively engaged in research on such subjects as collisions of heavy nuclei, statistical theory of nuclear reactions, multipole sum rules, isoinvariant RPA formalism, detailed evaluation of the charge-dependent contributions to nuclear forces, phenomenological explanations based on various nuclear models. Some nuclear research is also conducted in a few departments of physical chemistry, a good example being a phenomenological study of the correlation between the charge and mass distributions of fission products which has led to finding a discontinuity in the isotropic mass dispersion in various fission processes and was interpreted as a neutron shell effect.

\section{Plasma Physics}

This is practised on a very modest scale in the two research centres operated by the Atomic Energy Commission, where some applied nuclear physics work is also pursued. The research involves experiments with a condensed plasma focus, developing new sources and carrying on plasma diagnostics measurements. Turkey is participating in one of the European plasma physics projects, namely Textor (on the plasma-wall interaction).

\section{Applied Physics and Physics Engineering}

In order to attract more people to work on applied physics problems in industry, a degree of "Physics Engineering" was instituted about 20 years ago at the University of Ankara. Some universities followed suit and a few hundred physics engineers have graduated since then. Other universities preferred to grant degrees only in applied physics, so that we have now applied physicists and physics engineers working together in industry and various Government institutions, mainly on development and application problems in the fields of quality control, instrumentation, automation, data processing, nuclear energy, solar energy, health physics. However, it would be fair to say that the influence of physics in industry has still not reached an appreciable level.

\section{EPS General Conference}

I wish to end this short review by addressing on behalf of the Turkish physicists a most cordial welcome to our European colleagues who come to Istanbul on the occasion of the Fifth General Conference. We are all looking forward to the opportunities of discussing our problems with them; we are sure that the Conference will give rise to many new cooperative projects and we memorable one in many respects. shall do our best to make the Conference a

\section{University of Geneva}

The Faculty of Science has an opening for an:

\section{Extraordinary Professor of Structural Crystallography}

Duties:

Courses on modern methods of structure determination including neutron diffraction;

Interaction with other groups to help on crystallographic problems: Direction of research and thesis work as well as administration.

Requirements: Ph.D. in physics or crystallography; several years research experience.

Application: To be sent before 30 November 1981, to:

Faculté des Sciences, Université de Genève,

20. Quai Ernest-Ansermet, $\mathrm{CH}-1211$ Genève 4 